\title{
Diagnostic tumor marker of asbestos-related mesothelioma
}

\author{
Okio Hino · Masahiro Maeda
}

Received: 10 August 2007/ Accepted: 6 September 2007/Published online: 28 February 2008

(C) The Japanese Society for Hygiene 2008

\begin{abstract}
Mesothelioma is an aggressive tumor arising from the mesothelium, and is usually associated with previous exposure to asbestos. The incubation period of the tumor may be described as 30-40 years, and the prognosis is dismal. We previously discovered the Erc (Expressed in renal carcinoma) gene in the Eker rat model (Tsc2 gene mutant) and it was a homolog of the human mesothelin/ MPF gene. We developed a novel ELISA system (N-ERC/ mesothelin). Recently, we found that N-ERC/mesothelin was a potentially useful diagnostic marker for asbestosrelated mesothelioma.
\end{abstract}

Keywords Mesothelioma A Asbestos .

ERC/mesothelin/MPF · ELISA · Tumor marker

\section{Introduction}

Mesothelioma is an aggressive tumor arising from the mesothelium, and is usually associated with previous exposure to asbestos. The incubation period of asbestosrelated mesothelioma is estimated to be approximately 30 40 years. Once mesothelioma has occurred, there is no effective treatment. There is therefore an urgent need to identify mesothelioma tumor markers and a method for the early diagnosis of mesothelioma using these markers.

O. Hino $(\bowtie)$

Department of Pathology and Oncology,

Juntendo University School of Medicine,

2-1-1 Hongo, Bunkyo-ku, Tokyo 113-8421, Japan

e-mail: ohino@med.juntendo.ac.jp

M. Maeda

Immuno-Biological Laboratories Co., Ltd,

Fujioka, Gunma, Japan
Recently, several enzyme-linked immunosorbent assay systems for ERC/mesothelin have been developed, and their usefulness as a diagnostic tool has been assessed and demonstrated, as reported here.

\section{Discovery of the Erc gene}

The Eker (Tsc 2 gene mutant) rat model of hereditary renal carcinoma (RC) is an example of Mendelian dominantly inherited predisposition to a specific cancer in an experimental animal [1]. Tsc2 is an "initial gene" of carcinogenesis and abnormal networks of gene expression should be involved in tumor formation. To search for such alterations, we identified the highly expressed genes in Eker RC as the $C 3$ gene encoding the third component of complement, the annexin II gene encoding the calpactin 1 heavy-chain, the $\operatorname{Erc}$ (Expressed in renal carcinoma) gene, and the fra- 1 gene encoding a transcriptional factor activator protein 1 (AP-1) [2].

\section{ERC/MPF/mesothelin}

After we determined the complete primary structure of rat Erc cDNA, it was shown that the putative rat ERC product exhibits identity with human megakaryocyte potentiating factor, MPF/mesothelin [3-5]. A database search revealed that this predicted amino acid from Erc cDNA had identities of 87.4 and $56.1 \%$, respectively, with the mouse and human mesothelin genes. Two hydrophobic regions, a putative signal peptide near the amino-terminus and a putative glycosylphosphatidylinositol anchorage sequence near the carboxy-terminus, have been identified in human $\mathrm{MPF} / \mathrm{mesothelin}$. A putative furin cleavage sequence was 
also conserved in rat ERC. Rat Erc and human mesothelin were localized in chromosomes 10q12-21 and 16p13.3, respectively, both of which coincided with the locus of the tumor suppressor gene, the Tsc2/TSC gene. Therefore, rat ERC and human mesothelin are functional orthologs, and henceforth this protein will be referred to as ERC/ mesothelin.

\section{Example of translational research}

The most important modalities for the diagnosis of mesothelioma are chest radiography, chest computed tomography (CT), and magnetic resonance imaging (MRI) of the chest. However, it can be difficult to make an early diagnosis of mesothelioma using currently available diagnostic imaging techniques, and the identification of mesothelioma tumor markers and a method for the early diagnosis of mesothelioma using these markers are urgently needed.

The ERC/mesothelin protein is present on the normal mesothelium, a membrane lining several body cavities, including the pleura, peritoneum, pericardium and tunica vaginalis. It could be speculated that mesothelioma derived from the mesothelium should demonstrate overexpression of ERC/mesothelin. We have thus succeeded in establishing specific antibodies against ERC/mesothelin, and after validation by immunohistochemical studies on diseased tissue of mesothelioma patients, an enzyme-linked immunosorbent assay (ELISA) system has been developed, the usefulness of which has been assessed and demonstrated as a diagnostic tool [6-10]. Indeed, this is a real-life example of translational research.

\section{ERC/mesothelin in mesothelioma patients}

The full length of the human mesothelin gene codes the primary product, a 71-kDa precursor protein. This can be physiologically cleaved by some furin-like proteases into a 40-kDa C-terminal fragment that remains membranebound, and a $31-\mathrm{kDa} \mathrm{N}$-terminal fragment which is secreted into the blood [11, 12]. The C-terminal 40-kDa fragment is referred to as mesothelin. In contrast, the $\mathrm{N}$ terminal $31-\mathrm{kDa}$ fragment is a secreted protein identified as MPF (Fig. 1). Henceforth, the gene product of mesothelin is referred to by name. In order to avoid confusion arising from the use of the term mesothelin, the term N-ERC/ mesothelin is used for the N-terminal $31-\mathrm{kDa}$ fragment and $\mathrm{C}-\mathrm{ERC} / \mathrm{mes}$ thelin for the C-terminal $40-\mathrm{kDa}$ fragment. We have developed a novel ELISA system for the detection of $\mathrm{N}-\mathrm{ERC} / \mathrm{mes}$ thelin in the serum of mesothelioma patients, and have begun to examine its clinical usefulness

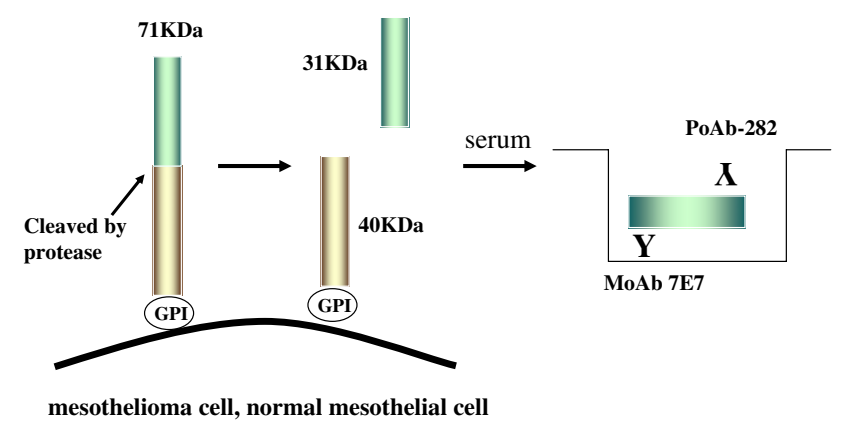

Fig. 1 Products of the Erc/mesothelin gene and the enzyme-linked immunosorbent assay (ELISA) system. The primary product of the Erc/mesothelin gene is a $71-\mathrm{kDa}$ precursor protein. This protein is physiologically cleaved, releasing its $31-\mathrm{kDa} \mathrm{N}$-terminal fragment into the blood. The authors' system detects $31-\mathrm{kDa}$ N-terminal fragments using a sandwich ELISA containing two antibodies against $\mathrm{N}$-terminal fragments [8]

[7]. Mouse monoclonal antibodies (clone 7E7) and rabbit polyclonal antibodies (PoAb-282) were raised against human N-ERC/ mesothelin and used to establish the ELISA system. Serum samples from seven patients with mesothelioma, four patients with carcinomatous pleuritis pleural metastasis (lung cancer), three with benign asbestos pleuritis, and 13 healthy volunteers were evaluated (Fig. 2). The diagnosis in patients with mesothelioma was confirmed immunohistochemically (all epithelial types).

So far, our ELISA system has detected much higher serum levels of $\mathrm{N}$-ERC/mesothelin in mesothelioma patients than in healthy controls or patients with other lung/ pleural diseases.

\section{Diagnostic markers of mesothelioma}

Recently Onda et al. [13] reported the generation of antiMPF monoclonal antibodies and the development of an ELISA for MPF (N-ERC/mesothelin). Their ELISA data showed that MPF levels were elevated in $91 \%$ of patients (51 of 56) with mesothelioma compared with healthy controls. Furthermore, MPF levels decreased in patients after tumor-debulking surgery. Thus, N-ERC/mesothelin may represent a promising tumor marker for mesothelioma.

So far, two groups have reported on ELISA systems for the detection of C-ERC/mesothelin. Robinson et al. have established an ELISA system for the detection of soluble mesothelin related protein (SMRP). In their first paper they assayed the serum concentrations of SMRP in serum samples obtained from 44 patients with histologically proven mesothelioma, 68 matched healthy controls, 40 of whom had a history of exposure to asbestos, and 160 patients with other inflammatory or malignant lung or pleural diseases [14]. They showed that $84 \%$ of the 44 mesothelioma patients had elevated serum concentrations 


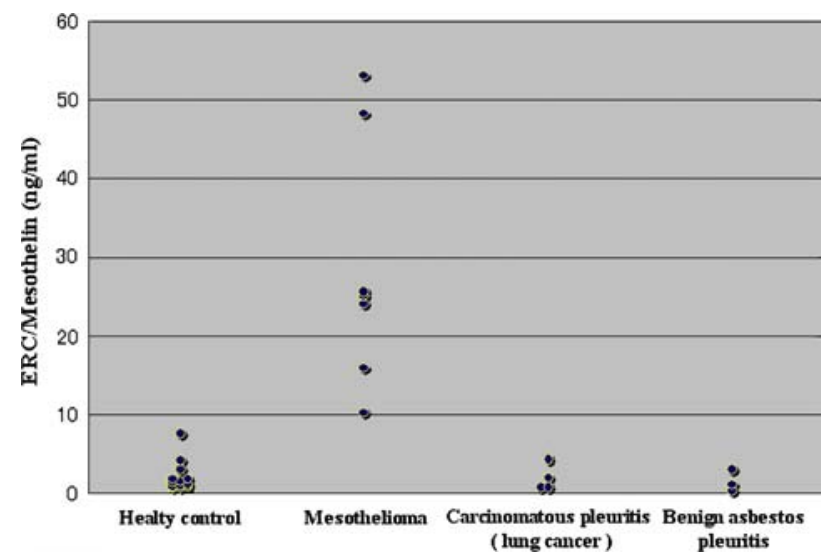

Fig. 2 Scatter plot of serum N-ERC/mesothelin. The serum concentrations of $\mathrm{N}-\mathrm{ERC} /$ mesothelin were much higher in mesothelioma patients than in the healthy controls, carcinomatous pleuritis (lung cancer) patients and benign asbestos pleuritis patients [5]

of SMRP, compared with $2 \%$ of the 160 with other lung/ pleural diseases. In their second paper they reported that measurement of the concentrations of SMRP in the serum as a marker of mesothelioma had a sensitivity of $83 \%$ and specificity of $95 \%$ for the diagnosis of mesothelioma in the first 48 mesothelioma patients examined [15]. Scherpereel et al. reported their data obtained using this ELISA kit (Mesomark; Fujirebio Diagnostic, Malvern, PA, USA) [16]. The mean serum SMRP level was higher in patients with mesothelioma $(2.05 \pm 2.57 \mathrm{nmol} / 1, n=28)$ than in those with pleural metastasis $(1.02 \pm 1.79 \mathrm{nmol} / 1, n=35)$ or benign lesions of the pleura $(0.55 \pm 0.59 \mathrm{nmol} / \mathrm{l}$, $n=28$ ). The pleural fluid SMRP concentrations were higher than the serum SMRP concentrations in all of the patient groups (mesothelioma, $46.1 \pm 83.2 \mathrm{nmol} / \mathrm{l}$; benign lesions, $\quad 6.4 \pm 11.1 \mathrm{nmol} / \mathrm{l}$; sis, $6.36 \pm 21.73 \mathrm{nmol} / \mathrm{l})$. Beyer et al. [17] reported analytical and preliminary clinical studies of MESOMARK ${ }^{\mathrm{TM}}$. They measured SMRP using this assay in 409 apparently healthy individuals, 177 patients with nonmalignant conditions, and 500 cancer patients, including 88 with malignant pleural mesothelioma (MPM). As a result, the mean SMRP was significantly higher in sera from patients with MPM $(7.5 \mathrm{nmol} / 1$; $95 \%$ confidence interval $[\mathrm{CI}]=2.8-12.1 \mathrm{nmol} / \mathrm{l} ; \quad n=88)$. SMRP was increased in 52 and 5\% of MPM patients and asbestos-exposed individuals, respectively. Hassan et al. have pursued the development of mAb against Erc/mesothelin. Some of these antibodies have been applied for the treatment of mesothelioma using immunotoxin technology [18].

Recently, a new mAb against C-ERC/mesothelin [19] and an ELISA system for the detection of C-ERC/mesothelin using these mAb have been produced, and they reported elevated serum levels of mesothelin in 40 of 56 (71\%) mesothelioma patients and 14 of 21 (67\%) ovarian cancer patients [20]. The serum levels of mesothelin were increased in $80 \%$ of patients who had positive tumor immunohistochemistry for mesothelin. They also observed a rapid decrease in the serum mesothelin levels after surgery in patients with peritoneal mesothelioma. These studies showed the possibility of diagnosis of mesothelioma.

Several ELISA systems for ERC/mesothelin have been developed and must be evaluated in parallel or systematic studies. Retrospective and prospective studies using these ELISA systems should be conducted in collaboration with immunohistochemical and imaging diagnosis.

\section{Conclusion}

We have started a large-scale prospective study on building construction workers, who run the risk of asbestos exposure, using our ELISA system. Considering the long incubation period of mesothelioma, the development of a system to maintain follow-up investigations for asbestosexposed individuals is required. Moreover, the formation of a network system of hospitals that take care of diagnosis and treatment in a comprehensive manner is necessary. At the same time, it is absolutely essential to resolve the mechanism of the occurrence of mesothelioma due to asbestos through basic research. The specific mechanism should throw new light on a definitive therapy for mesothelioma.

Acknowledgments This work was supported in part by Grants-inAid for Cancer Research from the Ministry of Education, Culture, Sports, Science and Technology of Japan, and the Ministry of Health, Labor and Welfare of Japan. Furthermore, this study was partially supported by a consignment expense of Molecular Imaging Program an "Research Base for PET Diagnosis" from the Ministry of Education, Culture, Sports, Science and Technology (MEXT), Government of Japan.

\section{References}

1. Eker R, Mossige JA. A dominant gene for renal adenomas in the rat. Nature. 1961;189:858-9.

2. Hino O, Kobayashi E, Nishizawa M, et al. Renal carcinogenesis in the Eker rat. J Cancer Res Clin Oncol. 1995;121:602-5.

3. Yamashita Y, Yokoyama M, Kobayashi E, Takai S, Hino O. Mapping and determination of the cDNA sequence of the Erc gene preferentially expressed in renal cell carcinoma in the Tsc2 gene mutant (Eker) rat model. Biochem Biophys Res Commun. 2000;275:134-40.

4. Yamaguchi N, Hattori K, Oh-eda M, Kojima T, Imai N, Ochi N. A novel cytokine exhibiting megakaryocyte potentiating activity from a human pancreatic tumor cell line HPC-Y5. J Biol Chem. 1994;269:805-8.

5. Kojima T, Oh-eda M, Hattori K, et al. Molecular cloning and expression of megakaryocyte potentiating factor cDNA. J Biol Chem. 1995;270:21984-90. 
6. Nakaishi M, Kajino K, Ikesue M, et al. Establishment of the enzyme-linked immunosorbent assay system to detect the amino terminal secretory form of rat Erc/Mesothelin. Cancer Sci. 2007;98:659-64.

7. Shiomi K, Miyamoto H, Segawa T, et al. A novel ELISA system for detection of a 'N-ERC/Mesothelin' in the sera of mesothelioma patients. Cancer Sci. 2006;97:928-32.

8. Maeda M, Hino O. Molecular tumor markers for asbestos-related mesothelioma: serum diagnostic markers. Pathol Int. 2006;56:649-54.

9. Maeda M, Hino O. Blood test for asbestos-related mesothelioma. Oncology. 2006;71:26-31.

10. Hino O, Shiomi K, Maeda M. Diagnostic biomarker of asbestosrelated mesothelioma: example of translational research. Cancer Sci. 2007;98:1147-51.

11. Chang K, Pastan I. Molecular cloning of mesothelin, a differentiation antigen present on mesothelium, mesotheliomas, and ovarian cancers. Proc Natl Acad Sci USA. 1996;93:136-40.

12. Chang K, Pai LH, Pass H, et al. Monoclonal antibody K1 reacts with epithelial mesothelioma but not with lung adenocarcinoma. Am J Surg Pathol. 1992;16:259-68.

13. Onda M, Nagata S, Ho M, et al. Megakaryocyte potentiation factor cleaved from mesothelin precursor is a useful tumor marker in the serum of patients with mesothelioma. Clin Cancer Res. 2006;12:4225-31.

14. Robinson BW, Creaney J, Lake R, et al. Mesothelin-family proteins and diagnosis of mesothelioma. Lancet. 2003;362:1612-16.

15. Robinson BW, Creaney J, Lake R, et al. Soluble mesothelin related protein: a blood test for mesothelioma. Lung Cancer. 2005;49:S109-11.

16. Scherpereel A, Grigoriu B, Conti M, et al. Soluble mesothelin related peptides in the diagnosis of malignant pleural mesothelioma. Am J Respir Crit Care Med. 2006;173:1155-60.

17. Beyer HL, Geschwindt RD, Glover CL, et al. MESOMARKTM: a potential test for malignant pleural mesothelioma. Clin Chem. 2007;53:666-72.

18. Hassan R, Bera T, Pastan I. Mesothelin: a new target for immunotherapy. Clin Cancer Res. 2004;10:3937-42.

19. Onda $M$, Willingham $M$, Nagata $S$, et al. New monoclonal antibodies to mesothelin useful for immunohistochemistry, fluorescence-activated cell sorting, Western blotting, and ELISA. Clin Cancer Res. 2005;11:5840-6.

20. Hassan R, Remaley AT, Sampson ML, et al. Detection and quantitation of serum mesothelin, a tumor marker for patients with mesothelioma and ovarian cancer. Clin Cancer Res. 2006;12:447-53. 